\title{
USING TEXTURE TO TACKLE THE PROBLEM OF SCALE IN LAND-COVER CLASSIFICATION
}

\author{
P. Corcoran*, A. Winstanley \\ National Centre for Geocomputation, National University of Ireland Maynooth, Co. Kildare, Ireland. \\ padraigc@cs.nuim.ie
}

KEY WORDS: land-cover classification, scale, texture, intensity, segmentation, visual perception.

\begin{abstract}
:
This paper discusses the problem of scale in current approaches to Object Based Image Analysis (OBIA), and proposes how it may be overcome using theories of texture. It is obvious that aerial images contain land-cover that is textured and thus any features used to derive a land-cover classification must model texture information and as well as intensity. Previous research in the area of OBIA has attempted to derive land-cover classification using intensity features only, ignoring the presence of texture. This has led to a number of issues with the current theory of OBIA. Using only intensity it is impossible to perform segmentation of textured landcover. In an attempt to tackle this problem it has become practice in OBIA to run segmentation at a number of different scales in the hope that each textured region will appear correctly segmented at some scale.

This process of performing segmentation at multiple scales is not in line with current theories of visual perception. Julesz (Julesz 1983) states that when we view an object our aperture is adjusted to view that object in its true form. Also in theories of visual object recognition each object or feature is represented only once in its true form. The result of integrating segmentation at multiple scales is the generation of a land-cover hierarchy in a bottom-up manner but this is not how our visual system generates such hierarchies. This process in the visual system is conversely very top-down, with the aggregation of objects not only being driven by their relative intensity or texture features but also our knowledge, desires and expectations. Quantitative evaluation is also made increasingly difficult due to the lack of ground truth for each scale; it is impossible to predict the appropriate appearance of ground truth at each scale. Given the fact that each land-cover is represented at a number of scales, the number of context relationships between objects which must be managed is exponentially large. This makes the task of deriving land-use from landcover increasingly difficult. If a robust set of intensity and texture features can be extracted and integrated correctly it would be possible to represent each land-cover in its true form within the one segmentation. Using a non-linear diffusion process and a geostatistical feature extraction algorithm we extract a set of intensity and texture feature respectively. Theses features are then integrated in such a manner to perform discriminate land-cover based on intensity where possible and texture where not. The motivation being that intensity features do not suffer from the uncertainty principle unlike texture thus giving more accurate boundary localization.
\end{abstract}

\section{INTRODUCTION}

Given the failings of traditional pixel based remote sensing techniques to provide accurate land-use classification of high resolution data in urban areas, many authors are attempting to move to a model of classification motivated by the human visual system (Blaschke and Hay 2001). This new approach to land-use recognition known as object based image analysis (OBIA) comprises two steps. The first is a land-cover segmentation of the scene, and this in turn is used as input to a structural pattern recognition system which models the structural relationships of individual land-cover to define landuse. It is generally accepted that the human visual system is in some form object based. Watt (Watt 1995) argues that this provides an alternative for the visual system to the computation of a full representation of spatial relationships within an image. The work presented in this paper focuses on the first of the above steps in OBIA, generating accurate land-cover segmentation in urban areas. We propose a new model of landuse classification which overcomes some issues with previous approaches.

The outline of this paper is as follows. Section 2 discusses some problems of multiscale representation and how they may be overcome by incorporating texture information.
This is followed by our methodology section which describes the algorithm developed to implement this theory. Results are reported and discussed in section 4 . Finally section 5 describes the conclusions drawn from this work.

The data used in this study consisted of subsets of orthorectified scanned colour aerial photography of Southampton city obtained from the Ordnance Survey Southampton, with a ground sample distance of $25 \mathrm{~cm}$. The photographs were converted from colour to greyscale and all analysis was performed on these greyscale images.

\section{PROBLEMS OF AND SOLUTIONS TO MULTISCALE REPRESENTATION}

Most previous work in the area of OBIA has attempted to derive land-cover segmentation using the visual cue of colour/intensity solely. This would be appropriate if all land-cover was completely uniform intensity but this is not the case. Most landcover contains small scale texture which must be taken into account when performing segmentation because attempting to perform accurate segmentation of textured land-cover using just intensity information is not possible. In an attempt to lesson this deficiency much effort has been invested into approaches which run intensity segmentation at multiple scales in the hope that

\footnotetext{
* Corresponding author.
} 
each land-cover will appear correctly segmented at some scale. All segmentations are then merged resulting in the generation of a land-cover hierarchy. This above process of running intensity based segmentation at multiple scales thus representing each land-cover a number of times, followed by the integration of results to form a land-cover hierarchy, is not in line with current theories of visual perception.

The representation of land-cover a number of times is not an accurate model of human visual system for a number of reasons. Julesz renowned for his work on the visual perception of texture states that "the aperture of attention changes its spatial scale according to the size of the feature being sought" (Julesz 1983), so when viewing a particular feature or object we observe it as one whole. Therefore when an area of uniform intensity or texture, which is a definition of land-cover, is viewed our aperture is adjusted to view it as one whole object. Also the principle of uniform connectedness (Palmer and Rock 1994) states that connected regions of uniform visual properties (e.g. intensity, texture) tend to be perceived initially as single objects and correspond to entry level units of visual stimuli. In theories of object recognition such as Biederman's recognition-by-components (Biederman 1987), complex objects are described as spatial arrangements of basic component parts. The first step is the segmentation of the image into a number of parts that can then be matched against representations of primitive components. In this theory each component which corresponds to land-cover in our problem is only represent once in its true form.

Integration of intensity based segmentation at multiple scale results in the generation of a land-cover hierarchy in a completely bottom-up (driven without knowledge) manner. Vecera and Farah (Vecera and Farah 1997) showed that the aggregation of objects in the human visual system to produce segmentation of a larger scale in the hierarchy is in fact topdown (knowledge driven), with the aggregation of objects not only being driven by their relative intensity or texture but also familiarity. They concluded, "top-down activation can partly guide the segmentation process". Pylyshyn (Pylyshyn 1999) also showed visual perception to be influenced by top-down factors.

The current approaches to OBIA not only raise theoretic issues but also raise some practical issues too. Running intensity based segmentation at a large number of scales in an attempt to best represent each land cover leads to high space complexity. For example Hay (Hay, Blaschke et al. 2003) used Gaussian scale space theory to represent image intensity features at 200 different scales. This process of representation at a number of scales also leads to large time complexity. If land-cover is represented once at its intrinsic scale then there exists one context relationship between each land-cover pair. On the other hand if each land cover is represented $n$ times this gives $n^{2}$ context relationships between each land-cover pair which must be managed. Choosing a subset of relationships in an attempt to reduce this complexity is also problematic.

With segmentation being run at a run of scales quantitative evaluation is also made increasingly difficult due to the lack of ground truth for each scale; it is impossible to predict the appropriate appearance of ground truth at a given scale. Therefore the only evaluation possible is qualitative performed through visual inspection. We agree with McCane (McCane 1997) that a human being is not the best judge to evaluate the output of any segmentation algorithm. In our model each land-cover is only represented one in its true form within one segmentation making quantitative evaluation possible.
Quantitative evaluation based on the performance of the system using the segmentation could be performed, but this does not give a good measure of segmentation performance but more the overall system.

A conceptual model which more accurately represents the human visual system and overcomes the above problems would possibly contain the following steps:

1. First a bottom-up driven land-cover classification must be performed where each land-cover is represented in its whole form within a single representation.

2. Generation of land-cover hierarchy which is both topdown and bottom-up driven.

3. Structural pattern recognition system which models the spatial relationships of different land-cover hierarchies to define land-use.

There are numerous more sophisticated models of human visual perception in literature and we certainly do not claim ours to be entirely accurate. Nevertheless we do believe it to be a more accurate model then the one used in current theories of OBIA. Hodgson (Hodgson 1998) describes an alternative conceptual model of human image interpretation for remotely sensed imagery using theories of human visual search.

In this paper we provide a methodology to perform the first of the above steps; producing accurate landcover segmentation. Land-cover may be defined as the "the description of the physical nature of the land-surface" (Wyatt, Greatorex-Davies et al. 1993), which could also be interpreted as areas of uniform intensity or texture. Hence if an accurate set of intensity and texture features are extracted and integrated correctly prior to segmentation being performed, it would be possible to perform segmentation where each land-cover is represented once in its true form within one representation.

\section{METHODOLOGY}

In the previous section we hypothesised that a system that generates land-cover segmentation incorporating both intensity and texture information could represent each land cover in its true form overcoming the above problems of multiscale representation. In this paper we implementation such a system using the following steps:

1. Extraction of intensity features.

2. Extraction of texture features.

3. Integration of intensity and texture features.

4. Segmentation performed using integrated features.

We now discuss each of these steps in more detail.

\subsection{Extraction of Intensity Features}

Intensity is an important feature when trying to discriminate between different land-covers. Due to within class variation or texture the original intensity values from remotely sensed images cannot be used directly as features because doing so would lead to over and under segmentation. A common approach to remove such variation is to perform linear diffusion which is equivalent to Gaussian smoothing. Linear diffusion is performed on image $I$ using to following equation

$$
\partial_{t} u_{i}=\operatorname{div}\left(\nabla u_{i}\right)
$$


with initial condition $u(x, y, 0)=I(x, y)$. This does indeed remove such within class variation but at the cost of giving mixed classes at land-cover boundaries and a loss in boundary localization. To overcome these failing a non-linear diffusion technique may be used (Perona and Malik 1990), where the amount of diffusion performed at any point is proportional to the gradient magnitude at that point

$$
\partial_{t} u=\operatorname{div}(g(|\nabla u|) \nabla u)
$$

Where $\mathrm{g}$ is a decreasing function of the form (Perona and Malik 1990)

$$
g(|\nabla u|)=e^{\left(-(|\nabla u| / K)^{2}\right)} .
$$

$K$ is a parameter which specifies the amount of smoothing an edge receives given a particular gradient magnitude. The motivation for using non-linear diffusion is that land-cover boundaries tend to have significantly lager gradient magnitude then the gradient magnitude due to the within class variation of texture and will hence receive less smoothing. Whitaker (Whitaker and Pizer 1993) showed that applying such a diffusion process to a texture image will not only preserve edges due to land-cover boundaries but also some edges due to texture. Obviously we only want to preserve edges due to landcover boundaries. Whitaker showed that smoothing the image with a Gaussian prior to computation of gradient magnitude will achieve this desired result. Incorporating this prior smoothing the non-linear diffusion equation now becomes

$$
\partial_{t} u=\operatorname{div}(g(|\nabla G(s(t)) * u|) \nabla u)
$$

where $G$ is a Gaussian function which takes one parameter sigma, which specifies the width of Gaussian. $s(t)$ is a linear decreasing function given by

$$
s(t)=s(t-1) r
$$

where is $0<r<1$. Unwanted edges diminish more rapidly then the edges due to land-cover boundaries. That is, gradient measurements become more reliable as the process evolves; hence a Gaussian of linear decreasing width is used.

Deng (Deng and Liu 2003) showed that applying a non-linear diffusion process to a textured image causes all regions to approach their average value. This is known as the diffusion property of average grey level invariance. If two land-covers are textured but have significant different average intensity values, applying non-linear diffusion will cause each land-cover to approach its average value. Applying land-cover classification now to the diffused image will give an accurate classification; an example of this is given in figure 1.

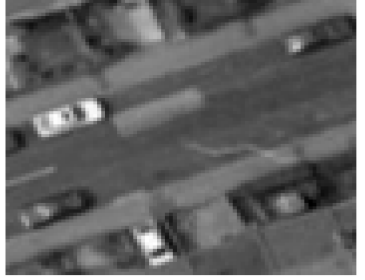

(a)

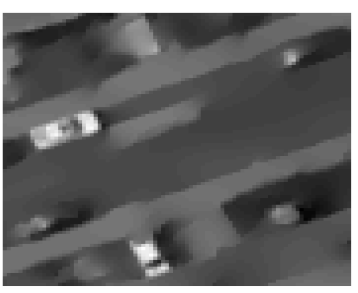

(b)
Figure 1: The footpaths in (a) have significant different average intensity values then neighbouring landcovers. Therefore accurate classification of these can then achieved using the diffused image in (b). Ordnance Survey (c) Crown Copyright. All rights reserved.

If two land-covers sharing a boundary have different textures but similar average intensity values, applying non-linear diffusion will cause these regions to be merged and classification based on the diffused intensity values will fail. In such a case it necessary to perform discrimination based on texture features and not the average intensity of land-cover. An example of this is shown in figure 2 .

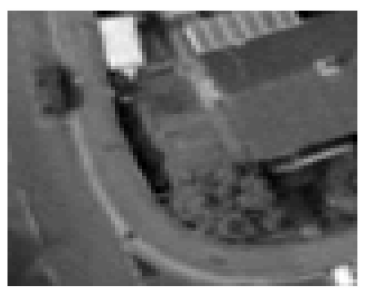

(a)

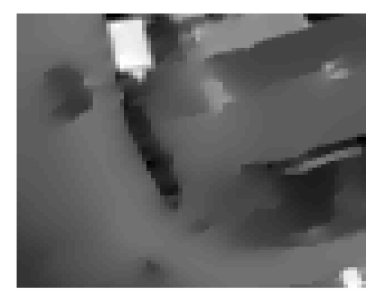

(b)
Figure 2: The building roof and the tree located just below in (a) have different textures but similar average intensity values. The diffused image in (b) shows that discrimination cannot be performed based on average intensity values. Ordnance Survey (c) Crown Copyright. All rights reserved.

In his implementation, Deng used an annealing diffusion function instead of smoothing with a variable width Gaussian prior to gradient magnitude computation. Both approaches are based on the same idea of performing linear diffusion prior to non-linear diffusion and would achieve similar results.

\subsection{Extraction of Texture Features}

As was shown earlier in cases where land-covers share similar average intensity values but different textures, incorporation of texture information is needed in order perform accurate landcover classification. For texture feature extraction a robust estimate of spatial autocorrelation or variogram known as the mean square-root pair difference (SRPD) is used (Cressie and Hawkins 1980)

$$
\operatorname{SPRD}(h)=\frac{1}{N_{h}}|z(x)-z(x-h)|
$$

Where $z$ represents pixels within the moving window, $h$ a vector in both direction and length and $N_{h}$ the number of pairs used to calculate the estimate. The SPRD measure of spatial autocorrelation has previously been shown to be an accurate model of texture (Lark 1996). In this paper the SPRD is 
calculated within a moving window using an isotropy vector of length 1 which returns a single feature image. It is obvious that texture has multi-scale and anisotropy properties but the purpose of this work is not to provide a completely accurate model of texture. Using a single feature image makes the algorithm more transparent and easier to visualise.

In their raw form the texture feature image is not suitable for classification. The texture features display significant within class variation which would lead to oversegmentation. Also the feature extraction algorithm responds strongly at land-cover boundaries when the moving window contains more then one class, this leads to doubly detected boundaries in classification (Martin, Fowlkes et al. 2004). To remove the within class variation while maintaining boundary localization the non-linear diffusion process discussed in section 3.1 is applied. A common approach in computer vision to removing the response at class boundaries is to apply separable median filtering. Separable median filters tend to preserve step discontinuities better then 2D median filters (Lim 1989).

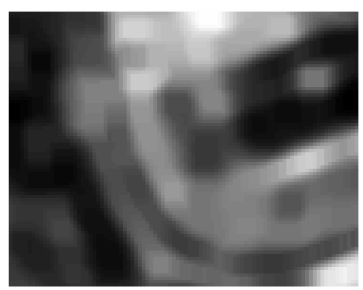

Figure 3: texture feature image extracted from figure 2(a). This feature provides better discrimination between building and tree then average intensity value shown in figure 2(b)

\subsection{Integration of intensity and texture features}

Traditional most remote sensing literature has integrated texture and intensity features in an unintelligent manner, simply joining feature vectors to form a single larger vector (Carr 1996). A number computer vision papers have shown that these features must be integrated in a more sophisticated manner in order to achieve accurate segmentation. Martin (Martin, Fowlkes et al. 2004) approached the problem as a supervised learning problem, where combination rules were learned from ground truth. A number of authors have tackled the integration task by defining a measure of texturedness and using this to modulate texture and intensity gradients. That is one cue will become disused in locations where the other cue should be operating (Malik, Belongie et al. 2001), (O'Callaghan and Bull 2005). The problem with this approach is that it is difficult to specify relatively how textured each land-cover is. Most research in remote sensing involving texture aims to provide the optimal set of features for discrimination, not to measure how strongly each land-cover is textured relative to each other. For any given set of features a new algorithm is needed to be derived to convert these to a measure of relative texturedness. In this paper we propose a new algorithm which does not suffer this failing. It is based on the principle that, discrimination between neighboring land-covers is performed using intensity where possible, that is when the land covers in question have a significant different average intensity values. In cases where this is not possible and land-covers have similar average intensity values but different textures, texture based discrimination is used. The uncertainty principle tells us that for any neighborhood property we cannot simultaneously measure that property and obtain accurate localization (Petrou and Sevilla 2006). Therefore for any texture feature extraction there is a tradeoff between size of window used to calculate this feature and accurate boundary localization. Intensity is not a neighborhood property consequently does not suffer this tradeoff and thus offers superior boundary localization. If two neighboring land-covers can be discriminated using either texture or intensity, discrimination should be based on intensity ignoring texture because this cue offers the superior boundary localization.

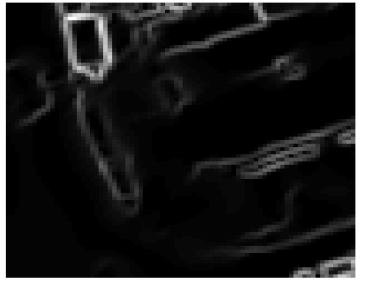

(a)

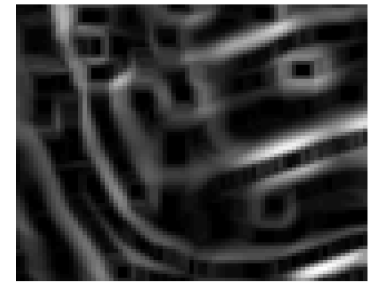

(b)
Figure 4: the gradient magnitude for figure 2(b) is shown in (a), this shows the location of edges in the diffused intensity image.

The gradient magnitude for figure 3 is shown in (b), this represents the location of texture edges.

To implement this method first we first calculate the gradient magnitude and orientation images for intensity and texture features. Each gradient magnitude image is normalized to a similar range to prevent one feature image from dominating. For each texture gradient magnitude value, we suppress the magnitude if by looking at the intensity gradient magnitude image we register that there is an intensity edge which corresponds to the same boundary which caused the texture edge. A texture and intensity edge corresponding to the same boundary will rarely spatially coincide due to the uncertainty principle so it is important to look in a neighborhood when trying to match these boundaries. The neighborhood of a location in the intensity gradient magnitude image is defined by placing a Gaussian shaped function with centre value of one centered over the location. Relative direction of each edge is also another important property to take into account when matching edges, if two edges have very different orientations then the probability of them belonging to the same boundary is low.

For each location in a given texture gradient magnitude image, the maximum intensity gradient magnitude value is calculated in the neighborhood of that location, giving more weight to edges of a similar direction and spatial location as follows

$$
\begin{aligned}
& \operatorname{IMM}(x, y)=\max _{u, v}(G(x-u, y-v) \cdot \operatorname{IM}(x-u, y-v) . \\
& |\cos (I O(x-u, y-v))-\cos (\operatorname{TO}(x, y))|)
\end{aligned}
$$

Where IMM represents the maximum intensity gradient magnitude in a neighborhood centered on $(x, y)$ we seek. $\mathrm{G}$ is a Gaussian shaped function with centre value of one centered on $(\mathrm{x}, \mathrm{y}), \mathrm{IM}$ is the intensity gradient magnitude image, IO is the intensity gradient orientation and TO the texture gradient orientation. The factor returned from the absolute operator in equation 7 is 1 if the texture gradient orientation and intensity gradient orientation are equal. This factor decreases as the angle difference increases reaching a minimum of zero when the two orientations are orthogonal (Grigorescu, Petkov et al. 2004). Using this measure of maximum intensity gradient magnitude in a neighborhood, we convert this value to a probability like measure using

$$
P I E=\exp (-I M M / p),
$$


where $\mathrm{p}$ is a user set parameter. Given a texture edge, PIE represents a probability like measure between zero and one of not being able to detect this boundary using an intensity edge instead. If a texture edge has a corresponding intensity edge this value will be close to zero, on the other if a texture edge does not have a corresponding intensity edge this value will be close to one. A texture edge in the presence of a corresponding intensity edge is now suppressed using

$$
T M=T M . P I E .
$$

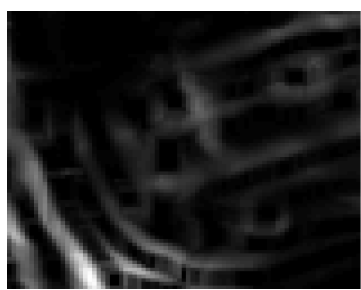

Figure 5: the suppression of texture gradient magnitude show in figure 4(b) using the intensity gradient magnitude of image of figure 4(a).

The intensity gradient magnitude and texture gradient magnitude images are now combined to form a single gradient magnitude image. For each location the maximum magnitude of all images is calculated and this value is used for the combined image.

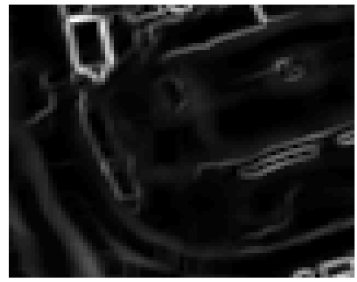

Figure 6: combined intensity and texture gradient magnitude images of figure 4(a) and figure 5 respectively.

Given a single gradient magnitude image produced using the above process of combining texture and intensity gradient magnitudes, segmentation is produced by apply the marker controlled watershed transform (Vincent and Soille 1991). The markers are defined using the extended-minima transform (Gonzalez, woods et al. 2003).

\section{EVALUATION}

This section provides a qualitative evaluation of the proposed algorithm. First we evaluate segmentation attained via integrated intensity and texture cues against the performance of segmentation derive solely from diffused intensity.

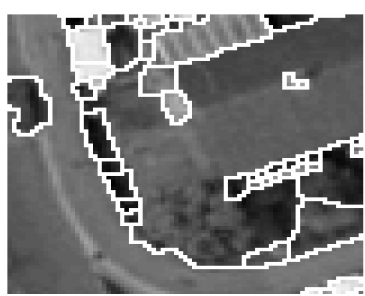

(a)

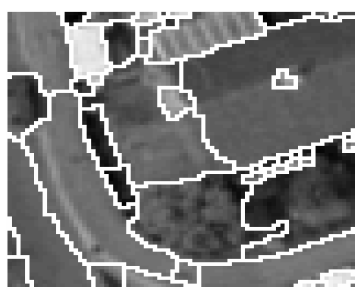

(b)
Figure 7: segmentation of figure 2(a) derived using only diffused intensity is shown in (a) while segmentation derived using integrated intensity and texture cues is shown in (b). Ordnance Survey (c) Crown Copyright. All rights reserved.

Figure 7 (a) shows that land-covers sharing boundaries, which have different textures but similar average intensity values are classified as single larger segments when texture information is not considered. Integration of texture information using the above methodology allows accurate classification of these landcovers which have a texture boundary but no intensity boundary figure 7(b). From figure 7(b) it can be also seen that a loss in boundary localization of intensity boundaries is not suffered.

Figure 8 shows the fusion of texture boundaries and intensity boundaries without previously suppressing the texture boundaries in the presence of intensity boundaries. Although all boundaries are present, we suffer a loss in localization of intensity boundaries.

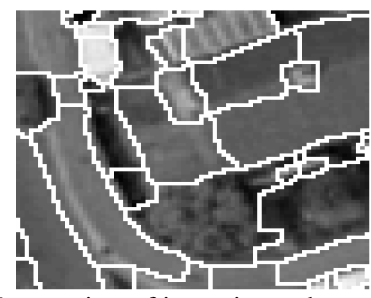

Figure 8: Integration of intensity and texture gradient magnitudes without prior suppression of texture gradient magnitudes. Ordnance Survey (c) Crown Copyright. All rights reserved.

Another qualitative evaluation example is shown in figure 9.

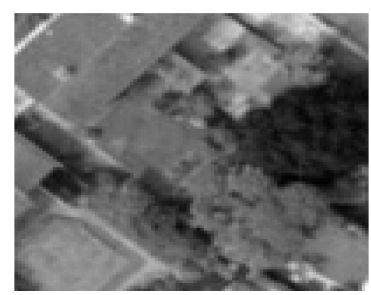

(a)

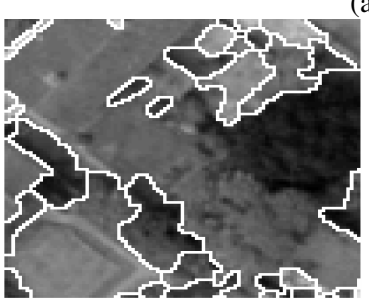

(b)

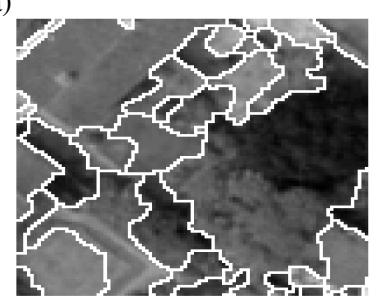

(c)
Figure 9: the diffused intensity based segmentation of (a) is shown in (b). The integrated intensity and texture segmentation is shown in (c). Ordnance Survey (c) Crown Copyright. All rights reserved. 
This qualitative evaluation shows the potential of the proposed algorithm to produce accurate land-cover segmentation incorporating texture and intensity information. It was found that the algorithm did not perform accurately on all test images so work needs to be done to improve the robustness of our approach. Integration of color information along with intensity and texture would also significantly improve performance. When performing evaluation, quantitative as well as qualitative evaluation needs to be performed. At this moment there does not exist a public available benchmark or database of remotely sensed images in urban areas and their corresponding ground truths such as the Berkeley segmentation dataset and benchmark (Martin, Fowlkes et al. 2001) for natural scenes.

\section{CONCLUSIONS}

Generating accurate land-use classification in urban areas from remotely sensed imagery is a challenging problem. The visual system can perform this task quite easily so a solution to the problem would be to model this system. Previous approaches in OBIA have been based on a conceptual model of visual perception which we believe is not entirely accurate. In this paper a new model which we believe is more in line with theories of visual perception is proposed. This model contains a number of steps, the first of these being a land-cover classification where land-cover is defined as areas of uniform texture and/or intensity. In this classification each land-cover is represented once in its whole form within one representation.

A novel segmentation algorithm which combines the cues of intensity and texture is proposed. It is based on the principle that boundary detection between classes should be performed using intensity where possible because intensity provides superior boundary localization over texture. The algorithm is still work in progress and when completed we hope to benchmark it against the Berkeley segmentation benchmark.

\section{REFERENCIES}

Biederman, I. (1987). Recognition-by-components: a theory of human image understanding. Psychological review 94: 115-147.

Blaschke, T. and G. Hay (2001). What's wrong with pixels? Some recent developments interfacing remote sensing and GIS. GIS - Zeitschrift fur Geoinformationssysteme 14(6): 12-17.

Carr, J. R. (1996). Spectral and textural classification of single and multiple band digital images. Computers and Geosciences 22(8): 849-865.

Cressie, N. and D. M. Hawkins (1980). Robust estimation of the variogram. Mathematical Geology 12: 115-125.

Deng, H. and J. Liu (2003). Development of anisotropic diffusion to segment texture images. Journal of electronic imaging 12(2): 307-316.

Gonzalez, R., R. woods, et al. (2003). Digital image processing using matlab, Prentice Hall.

Grigorescu, C., N. Petkov, et al. (2004). Contour and boundary detection improved by surround suppression of texture edges. Image and vision computing 22(8): 609-622.

Hay, G. J., T. Blaschke, et al. (2003). A comparison of three image-object methods for the multiscale analysis of landscape structure. Photogrammetric engineering and remote sensing 57(5-6): 327-345.
Hodgson, M. E. (1998). What size window for image classification? a cognitive perspective. Photogrammetric engineering and remote sensing 64(8): 797-807.

Julesz, B. (1983). Textons, the fundamental elements in preattentive vision and perception of textures. Bell system technical journal 62: 1619-1645.

Lark, R. M. (1996). Geostatistical description of texture on an aerial photograph for discriminating classes of land cover. International Journal of Remote Sensing 17(11): 2115-2133.

Lim, J. S. (1989). Two-dimensional signal and image processing, Prentice Hall.

Malik, J., S. Belongie, et al. (2001). Contour and texture analysis for image segmentation. International Journal of Computer Vision 43(1): 7-27.

Martin, D., C. Fowlkes, et al. (2001). A database of human segmented natural images and its applications to evaluating segmentation algorithms and measuring ecological statistics. International conference on computer vision, Vancouver. 416-423.

Martin, D. R., C. C. Fowlkes, et al. (2004). Learning to detect natural image boundaries using local brightness, color, and texture cues. IEEE Transactions on Pattern Analysis and Machine Intelligence 26(5): 530-549.

McCane, B. (1997). On the evaluation of image segmentation algorithms. Digital image computing : techniques and applications, Auckland. 455-460.

O'Callaghan, R. J. and D. R. Bull (2005). Combined morphological-spectral unsupervised image segmentation. IEEE Transactions on Image Processing 14(1): 49-62.

Palmer, S. E. and I. Rock (1994). Rethinking perceptual organization: The role of uniform connectedness. Psychonomic Bulletin and Review 1(1): 29-55.

Perona, P. and J. Malik (1990). Scale-space and edge detection using anisotropic diffusion. IEEE Transactions on Pattern Analysis and Machine Intelligence 12(7): 629-639.

Petrou, M. and P. G. Sevilla (2006). Image processing: dealing with texture, John Wiley and Sons Ltd.

Pylyshyn, Z. W. (1999). Is vision continuous with cognition? The case for cognition impenetrability of vision perception. Behavioural and brain sciences 22: 341365.

Vecera, S. P. and M. J. Farah (1997). Is visual image segmentation a bottom-up or an interactive process? Perception \& Psychophysics 59(8): 1280-1296.

Vincent, L. and P. Soille (1991). Watersheds in digital spaces: an efficient algorithm based on immersion simulations. IEEE Transactions on Pattern Analysis and Machine Intelligence 13(6): 583-598.

Watt, R. J. (1995). Some speculations on the role of texture processing in visual perception. Early vision and beyond. T. V. Papathomas, C. Chubb, A. Gorea and E. Kowler, The MIT press.

Whitaker, R. T. and S. M. Pizer (1993). A multi-scale approach to nonuniform diffusion. CVGIP: image understanding 57(1): 99-110.

Wyatt, B. K., J. N. Greatorex-Davies, et al. (1993). Comparison of land-cover definitions, Cambridge: institute of terrestrial ecology. 